\title{
Research on the Availability Evaluation of Command Information System
}

\author{
Mu Xiaofeng ${ }^{1}$, Zhang Weiming ${ }^{1}$, Lin Peng ${ }^{2}$, Li Xiaoli $^{2 *}$ \\ ${ }^{1}$ Science and Technology on Information Systems Engineering Laboratory, National University of \\ Defense Technology, Changsha 410073, China \\ ${ }^{2}$ China Electronic Equipment of System Engineering Institute, Beijing 100141, China \\ Email: 13701123025@163.com
}

Keywords: availability of command information system, index construction principles, evaluation system

\begin{abstract}
Focused on the availability of command information system, the availability model of command information system is constructed from capability, business function, application process and application object firstly. Secondly, the availability index of command information system is established in principle. Then, the availability evaluation index framework of command information system is designed from requirement consistency, function conformance, operational applicability and user friendliness, which are analyzed in detail. Further, the data acquisition channels and calculation methods of specific index are described, which lays a foundation for the availability evaluation of command information system.
\end{abstract}

\section{Introduction}

Command information system is a complex system supporting command and control of the whole process of combat un- der the informatization condition, which has the characteristics of complex system structure, multiple functions, long online time and high reliability requirements. As the material basis of information warfare, command information system has the function of binder, multiplier and catalyst [1-2]. Therefore, it is very important to study the availability of command information system and analyze the main factors that affect system availability, and to optimize the adjustment of command information system in information warfare effectively.

System availability refers to the information system product- $\mathrm{s}$, which can successfully meet user needs when they are used, and the system availability can be understood and evaluated from system function and user experience [3]. In command information system, the availability refers to the ability to effectively support the whole process of command and control during combat. The availability is closely related to the overall function and the combat capability of command information system. It is an important guarantee for capturing information superiority, decision advantage and action advantage.

First of all, in order to solve the problem of the availability of command information system, the paper constructs the availability model from the four perspectives of capability requirements, business function, application process and application object [4-5]. Secondly, according to the 
characteristics of command information system, the principle of constructing the availability index is established. Finally, based on the avail- ability model and the index framework construction principle, this paper designs the availability evaluation index framework from requirement consistency, function conformance, operational applicability and user friendliness.

\section{Availlability Model}

Combined with the construction and organization of command information system analysis [6], the availability of command information system can be affected by the system itself and the system using the object factors. The availability model of command information system is constructed from capability, business function, application process and application object, as shown in Figure 1.

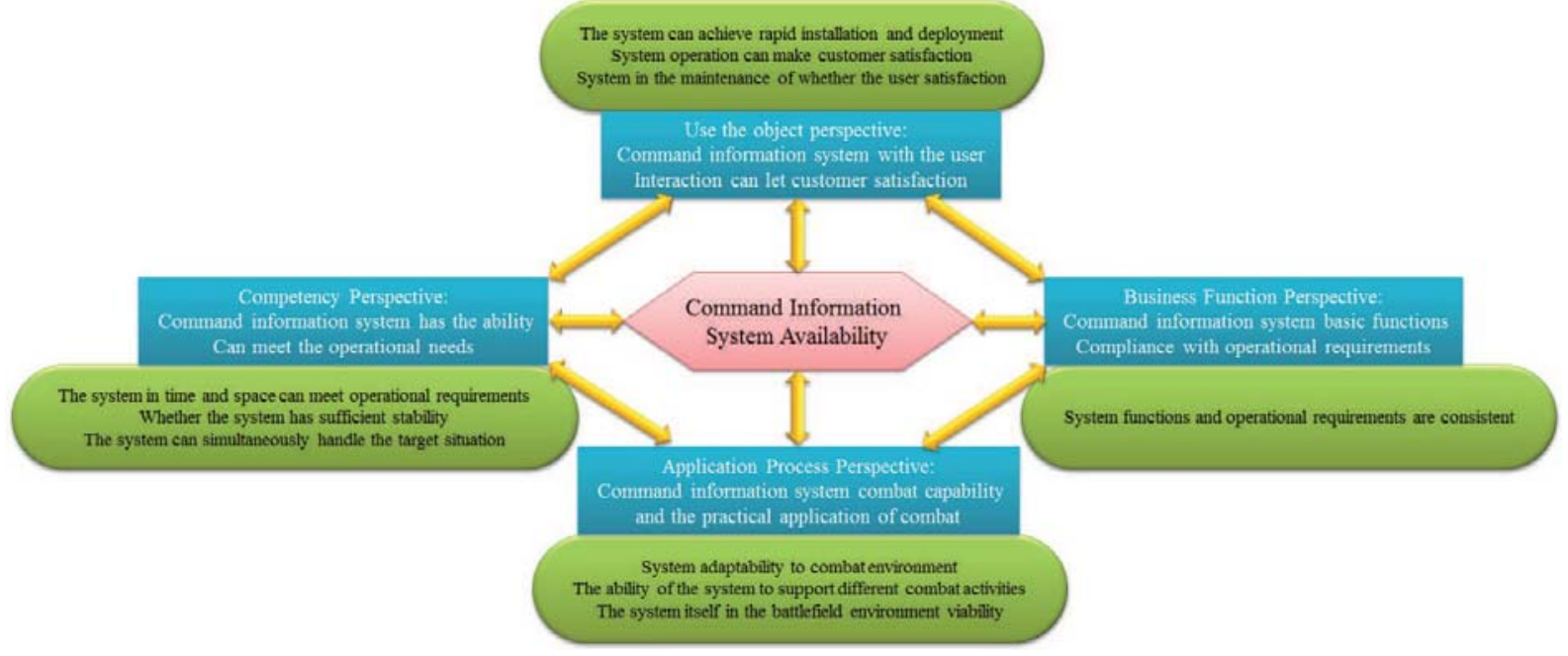

Fig. 1: Availability model of command information system.

- Capacity requirements perspective

Capability requirements perspective mainly assesses the consistent of command information system and operational requirements, including the scope of time and space, system reliability and stability, the performance of the system target processing, system interoperability and so on.

- Business function perspective

Business function perspective mainly assesses the basic function of command information system and the degree of compliance of the actual combat application. For example, the ability to obtain information to meet the operational requirements of intelligence information, the ability to support decision-making needs of the command and so on.

- Process perspective

The process perspective mainly assesses the extent and application of the combat effectiveness of command information system in practical combat applications. Including the ability of the system to adapt to the combat environment, the system support for different operational activities, the system itself in the battlefield environment, the viability and so on.

- Object perspective

The object perspective mainly assesses the user interaction process and the user's satisfaction. Mainly including system installation and deployment, system operation, system maintenance and other aspects of user satisfaction.

\section{Construction of Availability Index}

Command information system availability is a complex, comprehensive nature of many 
availability factors. In order to comprehensively and scientifically evaluate the availability of command information system, it is necessary to establish a set of evaluation index which can comprehensively reflect the essential characteristics and objective requirements of command information system according to certain theoretical requirements and principles. In the establishment of the availability index for command information system, some principles [3,7-8] should be followed.

- Scientific principles

The establishment of the availability index should conform to the characteristics of the construction and development of the military command information system, and the index is strict, the concept meaning is clear, not conflicting, scientific and reasonable.

- Systematic principles

The availability index should fully consider the influence factors of command information system, which can comprehensively, systematically reflect the availability essence of command information system.

- Principle of objectivity

The purpose of assessing the availability of command information system is to provide a reference for the operational application of command information system. Therefore, the evaluation index should be able to objectively and truthfully reflect the real availability of command information system.

- Principle of stability

The evaluation index should be able to ensure the stability and reliability of the whole evaluation process, which eliminate the influence of evaluation time and situation and ensure the validity of evaluation results.

- Principle of operability

There are many factors influencing the availability of command information system, and the differences are also great. The selection of evaluation index should be practical and convenient, so that the index framework can be easily obtained, quantified and operable.

- Hierarchical principle

The availability index of command information system should be divided into levels, each level set a number of index for evaluation. On the basis of ensuring the concise nature of the availability, the evaluation index framework is strengthened systemically and scientifically.

- Principle of conciseness

The evaluation index should be concise and avoid tedious in ensuring comprehensive and comprehensive reflection of the relationship between the evaluation object and the index.

\section{Design of Availability Evaluation Index}

Based on the characteristics of command information sys- tem and the availability model, according to the principles of scientific, systematic, objective, stable, operable, hierarchical and concise, various relevant influencing factors are screened and perfected. The availability evaluation index framework is decomposed from demand consistency, function conformance, operational suitability and user friendliness according to sys- tem availability requirements. It is divided into 15 secondary level index and 23 third level index, as shown in Figure 2. Through these specific evaluation index, the availability of command information system can be evaluated from all dimensions. The quantitative index and the qualitative index can be combined to ensure the objective, systematic and comprehensive, further, to ensure the scientifically, standardization and rationality of the availability evaluation index of command information system. 


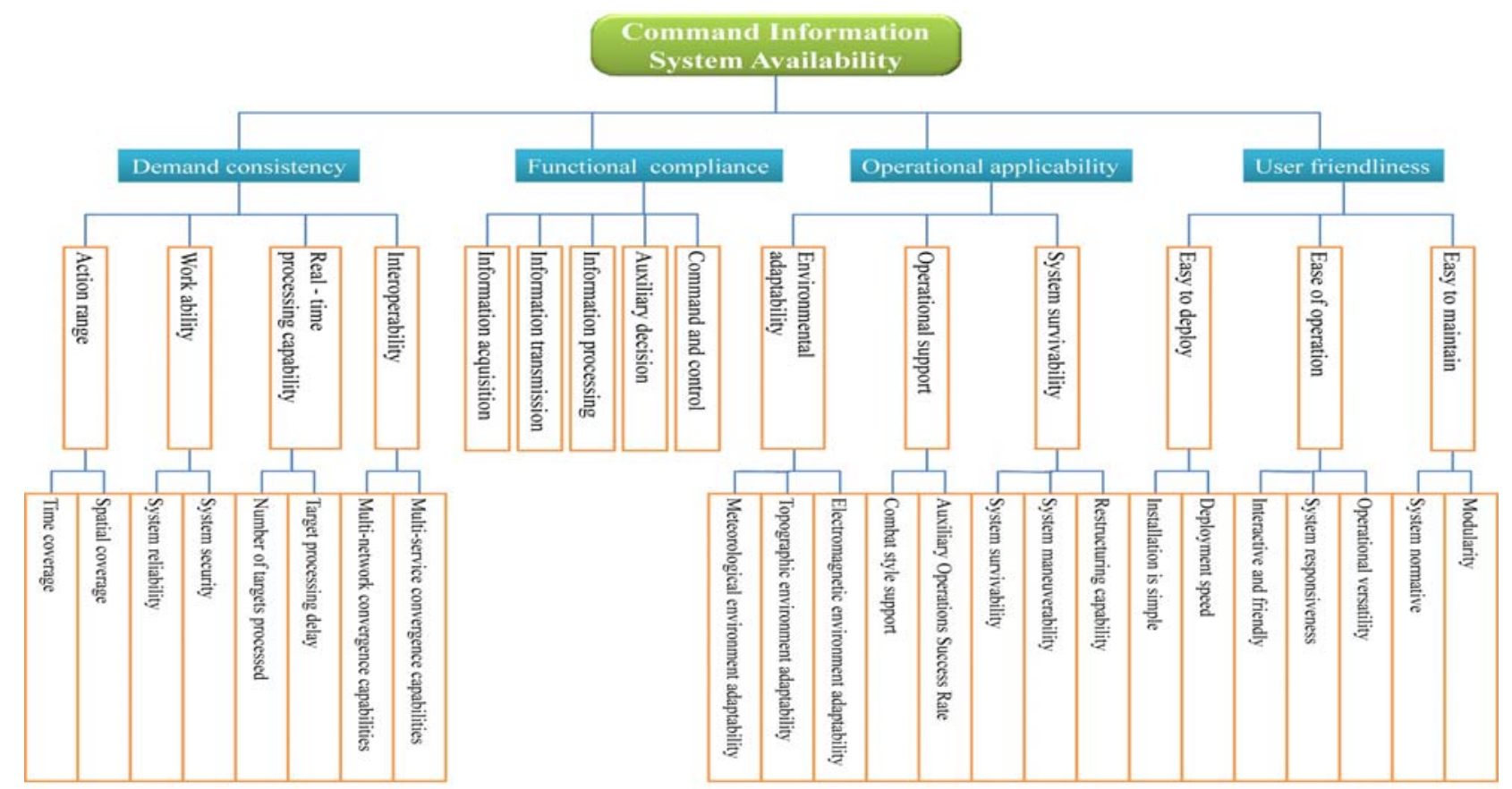

Fig. 2: Availability index of command information system.

\section{A. Demand consistency}

The demand consistency of requirements refers to the users' requirements and the ability of command information systems. Command information system capacity requirements include the scope of work, work capacity, real-time processing capabilities and interoperability and other content, as shown in Table 1.

Table 1: Requirements consistency evaluation index

\begin{tabular}{|c|c|c|}
\hline \multirow{2}{*}{ First level index } & Second level index & Third level index \\
\hline \multirow{7}{*}{ Demand consistency } & Range of action & $\begin{array}{c}\text { Time coverage, } \\
\text { spatial coverage }\end{array}$ \\
\cline { 2 - 3 } & Work ability & $\begin{array}{c}\text { System reliability, } \\
\text { system security }\end{array}$ \\
\cline { 2 - 3 } & Real-time \\
& processing capability & $\begin{array}{c}\text { Number of targets processed, } \\
\text { target processing delay }\end{array}$ \\
\cline { 2 - 3 } & Interoperability & $\begin{array}{c}\text { Multi-network convergence capabilities, } \\
\text { multi-service convergence capabilities }\end{array}$ \\
\hline
\end{tabular}

- Action scope

Scope of the performance of command information system is able to play a normal combat capability of time and space. To measure the time coverage, spatial coverage and other index, the specific information may be reflected in command information system to detect the early warning, command and control of the region, airspace, radius and time period.

- Work ability

Work ability mainly for command information system can play its combat effectiveness of the normal capacity. Usually, the system reliability, safety and other index can be used to evaluate. 
- Real-time processing capability

Real-time processing capability characterize the ability of command information system to track and process real-time targets, including the number of processing, delay and so on.

- Interoperability

Interoperability ability covers interconnection, interworking, interoperability, and involves multi-network integration, multi- service integration and so on.

B. Functional compliance

Functional compliance refers to the degree of conformance between operational requirements and the functions of command information system during the combat operations. The paper analyzes the conformance between the basic functions of command information system and the business function requirements, as shown in Table 2 .

Table 2: Functional conformity evaluation index

\begin{tabular}{|c|c|}
\hline First level index & Second level index \\
\hline \multirow{3}{*}{ Demand consistency } & $\begin{array}{c}\text { Information acquisition, } \\
\text { information transmission, } \\
\text { information processing, } \\
\text { auxiliary decision, } \\
\\
\text { command and control functions }\end{array}$ \\
\hline
\end{tabular}

Although different command information systems have different task objectives and application areas, they generally have some common functions, including information acquisition, information transmission, information processing, auxiliary decision, command and control functions.

C. Operational applicability

Operational applicability refers to the extent to which command information system meets the actual combat requirements in actual combat operations. This paper evaluates the operational adaptability of command information system from the three aspects of environmental adaptability, operational support and survivability, as shown in Table 3.

- Environmental adaptability

Environmental adaptability characterizes the adaptability of command information system to the environment in which it is deployed, including severe meteorological conditions, complex terrain and electromagnetic environments.

- System survivability

System survivability refers to the ability of command information system to maintain its normal work in the face of enemy's attack and the damage of some components in high combat environment. Such as survivability, mobility, dynamic reorganization capabilities, and so on. 
Table 3: Operational applicability evaluation index

\begin{tabular}{|c|c|c|}
\hline First level index & Second level index & Third level index \\
\hline \multirow{4}{*}{$\begin{array}{c}\text { Operational } \\
\text { applicability }\end{array}$} & $\begin{array}{c}\text { Environmental } \\
\text { adaptability }\end{array}$ & $\begin{array}{c}\text { Meteorological, } \\
\text { topography, } \\
\text { electromagnetic }\end{array}$ \\
\cline { 2 - 3 } & System survivability & $\begin{array}{c}\text { System survivability, } \\
\text { system maneuverability, } \\
\text { system restructuring }\end{array}$ \\
\cline { 2 - 3 } & Operational support & $\begin{array}{c}\text { Combat style support, } \\
\text { auxiliary operations success rate }\end{array}$ \\
\hline
\end{tabular}

- Operational support

The operational support mainly refers to the system support for the combat style, task success rate and so on. The support of the combat style, including air defense and anti-missile, urban operations, jungle combat, crossing the sea landing. Auxiliary combat success rate refers to the success of the combat mission to complete the target probability.

D. User friendliness

User friendliness reflects the level of friendliness of command information system. Command information system involves a wide range of users, uneven levels of the informatization, and different users habits. In order to better adapt to different users, command information system user-friendly mainly from the easy to deploy, easy to operate, easy to maintain and other aspects of evaluation, as shown in Table 4.

Table 4: User friendliness evaluation index

\begin{tabular}{|c|c|c|}
\hline First level index & Second level index & Third level index \\
\hline \multirow{3}{*}{ User friendliness } & Easy to deploy & $\begin{array}{c}\text { Installation is simple, } \\
\text { deployment speed }\end{array}$ \\
\cline { 2 - 3 } & Easy to operate & $\begin{array}{c}\text { Interactive and friendly, } \\
\text { system responsiveness, operational } \\
\text { versatility }\end{array}$ \\
\cline { 2 - 3 } & Easy to maintain & $\begin{array}{c}\text { System normative, } \\
\text { modularity }\end{array}$ \\
\hline
\end{tabular}

Easy to deploy

Easy to deploy refers to the system in the installation, deployment and other aspects of the ease. It is directly related to the time of system installation, speed of deployment and redeployment, and time.

- Easy to operate

Easy operation refers to the ease of operation of the system. Mainly reflected in the system for users to experience the use of the degree of satisfaction.

- Easy to maintain

Easy maintenance refers to the ease of system maintenance. It is mainly related to the normative nature of the system and the modularity of the system. 


\section{Conclusion}

This paper focuses on the construction of the availability evaluation index of command information system. Firstly, the availability model of command information system is constructed from capability, business function, application process and application object. Secondly, on the basis of analyzing the establishment principle of the availability index, the availability evaluation index of command information system is designed, and the connotation of each evaluation index is analyzed in detail. Finally, the data acquisition channels and calculation methods of specific index are expounded from the four dimensions of consistency of needs, functional compliance, operational applicability and user friendliness, which lays the foundation for the availability evaluation of the later implementation of command information system.

\section{References}

[1] Cao Lei. Command Information System [M]. National Defense Industry Press, 2012: 15-20.

[2] Ling Yanxiang, Chen Honghui. Command information system domain integration capability index system [J]. Journal of firepower and Command Control, 2013, 38(4): 9-11.

[3] Li Lin, Zhang Lingxian, Li Daoliang and etc. Selection model of suitability evaluation index system for greenhouse intelligent control system [J]. Transactions of the Chinese Society of Agricultural Engineering, 2012, 28(3): 148-153.

[4] Liu Guotai, Wang Ruihua, Liu Jingxu. Command information system capability requirement satisfaction evaluation model [J]. Journal of China Institute of Electronics and Information Technology, 2014, 9(6): 603-608.

[5] Liu Gang, Zou Zili, Hu Yanzhi and etc. Research on the guidance mode of operational effectiveness evaluation of command information system [J]. Journal of Ordnance Industry Automation, 2015, 34(7): 65-70.

[6] Jin Hong, Sun Zhengjie, Yu yue and etc. Composition and construction of command information system [J]. Journal of Information Systems Engineering, 2015, 27(3): 12-14.

[7] Zhang Xianzhi, Dan Wentao. Research on evaluation system of internal control of Chinese enterprises [J]. Journal of Auditing Research, 2011, 31(1): 69-78.

[8] Liu Gang, Yang Yingmei, Ma Wentao and etc. Construction and selection of high-tech park prediction index system [J]. Journal of Value Engineering, 2015, 24(3): 1-5. 